\title{
Research on the Direction of Ion Channel Related to Epileptic Seizures
}

\author{
Jianing Yin $^{1}$ \\ ${ }^{1}$ University of Jinan, School of Biological Science and Technology Shandong China, Jinan
}

\begin{abstract}
Epilepsy is a group of chronic brain diseases characterized by transient central nervous system dysfunction caused by repeated abnormal synchronization of neuronal discharges in the brain, with sudden onset and repeated seizures. Epilepsy has been listed as one of the five major neuropsychiatric diseases of the World Health Organization (WHO). Hereditary epilepsy refers to epilepsy syndromes previously classified as idiopathic generalized epilepsies (IGEs), which encompasses several different epilepsy syndromes ranging in clinical severity from relatively benign febrile convulsions (FS) and childhood absence epilepsy (CAE) to the more severe juvenile myoclonic epilepsy (JME) and generalized epileptic seizures with febrile convulsions (GEFS+). This article analyzes the direction of ion channel related to epileptic seizures. It will look forward to the future research direction of some of the ion channels related to epileptogenesis.
\end{abstract}

\section{INTRODUCTION}

The causes of epilepsy are complex, and what exactly happens to the brain during epileptogenesis is epilepsy is a hot topic of recent research. Ion channels are important physiological components related to the occurrence of neuronal excitations, usually consisting of several subunits, accompanied by the binding of auxiliary groups to complete their physiological properties. The complex composition of subunits and the variety of auxiliary groups regulate the excitation and inhibition of neurons, and their functional impairment may be one of the important factors leading to epilepsy. Much progress has been made in human research on epilepsy and ion channels, but there is still a long way to go to clarify the molecular mechanism of epileptogenesis and to prepare precise drugs for epilepsy. This article will look forward to the future research direction of some of the ion channels related to epileptogenesis.

\section{Mutations in the gene encoding the sodium channel affect epileptogenesis}

Take Nav1.1 as an example, from the current level of research, people are more interested in finding out whether Nav1.1 can cause epilepsy from the perspective of gene mutation, and what kind of gene regulates the process of epilepsy. The specific molecular mechanisms of Nav1.1 inactivation are less well understood by scientists, but of course, this falls within the realm of molecular and structural biology. After understanding the general relationship between Nav1.1 and SCN1A and epileptogenesis, attention has gradually turned to the specific molecular mechanisms of its dysregulation. Our gene sequencing technology is now so sophisticated that it may be relatively easy to determine whether a gene is mutated or not, and even more difficult to study the specific mechanisms by which the activity of a mutated expressed protein changes. But research is underway to determine the status of the molecule affected after a mutation, both at the gene level and at the protein itself.

\subsection{SCN1A Gene Mutations and Epilepsy}

The human sodium channel family includes seven neuronal channels that are critical for initiation and propagation of action potentials in CNS and PNS.SCN1A is part of SCN1A -SCN2A-SCN3A gene cluster on chromosome 2q24, which encodes the pore-forming subunit of the sodium channel. [1]

SCN1A encodes the alpha subunit of the voltage-gated sodium channel Nav1.1. Its 26 exons are distributed over $100 \mathrm{~kb}$ of genomic DNA. Genetic defects in the coding sequence result in generalized epilepsy with hyperthermia $($ GEFS +$)$ and a range of childhood epileptic encephalopathies of varying severity (e.g., SMEI). Statistically, there are more than 100 new mutations distributed throughout the gene. A number of mutant clusters have been observed in the $\mathrm{C}$-terminus and in the loop between segments 5 and 6 of the first three domains of the protein. Functional studies to date have not been able to demonstrate consistency between changes in channel characteristics and clinical phenotypes. However, of all known epilepsy genes, SCN1A is currently the most clinically relevant. It is by far characterized by the largest number of mutations associated with epilepsy[1].Clearly, the channel should be inextricably linked to the development of epilepsy. 
Table1. A variety of functional abnormalities in mutant alleles of SCN1A encoding the sodium channel Nav1.1[4]

$\begin{array}{ll}\text { Mutation } & \text { Major biophysical abnormalities observed in indicated experimental system } \\ \text { D188V } & \text { Decreased entry into and increased rate of recovery from slow inactivated state }(m)(40) \\ \text { T875M } & \begin{array}{c}\text { Enhanced entry into slow inactivated state }(X)(45) \text {; enhanced entry and hyperpolarizing shift into steady-state slow inactivation }(m) \\ (39,42) \text {; increased persistent current }(m)(39,42)\end{array} \\ \text { W1204R } & \begin{array}{l}\text { Hyperpolarized shift in voltage dependence of activation and inactivation }(X)(46) \text {; increased persistent current }(m)(39) \\ \text { Increased rate of recovery from fast inactivation }(X)(45) \text {; increased persistent current }(m)(39) \text {; slowed inactivation and } \\ \text { R1648H }\end{array} \\ \text { increased recovery from inactivation }(m)(41) \\ \text { R1648C } & \begin{array}{l}\text { Increased persistent current }(m)(33) \\ \text { I1656M }\end{array} \\ \text { G1674R } & \text { Nepolarized shift in voltage dependence of activation }(m)(43) \\ \text { D1866Y } & \text { Depolarized shift in voltage dependence of fast inactivation }(X) \text { due to reduced association with } \beta 1 \text { subunit }(m)(23)\end{array}$

The most prominent effects observed in each study are indicated; most mutations altered several biophysical properties of the channel. $m$, transfected mammalian cells; $X$, Xenopus oocyte system.

SCN1A mutations were first identified in families with mild hereditary generalized epilepsy with hyperthermia plus $(\mathrm{GEFS}+)$ [2], and shortly thereafter in patients with severe myoclonic infantile epilepsy (SMEI) (also known as Dravet syndrome) [3]. More than 650 SCN1A heterozygous mutations have been identified in patients with SMEI and approximately $10 \%$ mutations have also been observed in patients with GEFS + [4].

Andrew et al [5] identified the novel mutation W1204R and seven other coding changes by conformation-sensitive gel electrophoresis and manual sequencing of variants in 226 patients with juvenile myoclonic epilepsy, anaplastic epilepsy, or hyperthermic convulsions. Of the seven other coding changes, three of them were potentially pathogenic mutations at the location of the other seven coding changes. A SNP was found 18 bp upstream of the shear acceptor site in exon 3 in seven of these 226 patients, but not in 185 controls. These mutations included demethylation of certain $\mathrm{CpG}$ residues in the arginine codon and short-term repeats of simple sequences. The remaining mutations are missense mutations that may result in gain or loss of function [6].

From the above facts, it can be inferred that we already have some knowledge about the mutations in SCN1A gene. However, according to Meisler [6] et al. it is clear that SCN1A has a significant haploinsufficiency and was also found in the study by Martin [7] et al. In many patients with epilepsy who also have other genes that do function, the patients behave similarly to the epileptic onset in patients with loss of function of the SCN1A gene alone, but we have not made any further progress beyond the fact that SCN1A has a role in the activation of inhibitory neurons. Therefore, although sufficient research data are available to show that SCN1A mutations are associated with the onset of hereditary epilepsy, a more precise relationship between SCN1A mutations and epilepsy has not been described.

\subsection{Nav1.1 Dysfunction and Epilepsy}

In their study of the effects of the Dravet syndromeassociated NaV1.1 truncation mutation on the expression and function of sodium channel membrane proteins, Haiqing $\mathrm{Xu}$ [8] et al. investigated the pathogenic mechanism of the truncation of NaV1.1 and concluded that the expression of membrane proteins associated with the mutant type was significantly reduced compared to the wild type and the original function of the sodium channel was lost. In vitro electrophysiological testing of neuronal cells from patients expressing mutant Nav1.1 by Jingxin Liu [9] revealed that the mutation could be expressed normally in cells cultured in vitro, but with no or only minimal Na currents in the channel. From this, it can be tentatively determined that the mutation can alter the performance of neurons to produce action potentials. Miriam et al.[6] summarized several mouse models that have been developed with four null or missense mutations in SCN1A. Several null alleles are generated by directed mutagenesis on embryonic stem cells (ES). Among them, pure null mice develop spontaneous seizures and die in the third postnatal week. Heterozygous null mice also exhibit spontaneous seizures, but about $50 \%$ survive to adulthood. Significant reductions in current were observed in cortical neurons of -/- and +/- mice, mice carrying the GEFS + mutation $\mathrm{R} 1648 \mathrm{H}$, in inhibitory neurons, but not much in excitatory neurons. These facts and data allow to speculate that SCN1A encodes the sodium channel Nav1.1 which acts to activate inhibitory neurons, and the finding of heterozygosity for the GABAA receptor null allele in epileptic patients provides independent evidence for the critical role of inhibitory signals in epileptogenesis [10]. In contrast, SCN1A mutations reduce the inhibitory signal.

Miriam [11] et al. have suggested that the dependence of inhibitory neurons on the level of that particular sodium channel may be a consequence of their subcellular localization, such as their function in the axon start segment. However, people have not reported any relevant studies in this area. So how does normal SCN1A generate inhibitory signals? How do its substructure changes and auxiliary and small molecule modifications proceed? As we come to a conclusion about these issues, epilepsy treatment may also make a breakthrough at the same time. 


\section{HCN channels influence epileptogenesis}

Unlike Nav1.1, studies on HCN channels and epileptogenesis have been conducted more on the combination, structure, and function of channel subunits. On the other hand, the association between HCN channel mutations and epilepsy could not be confirmed because of insufficient data and lack of obvious association. It is also because the structure and function of $\mathrm{HCN}$ channels are unique, and many difficulties have been encountered in the study of their structure and function; the functions of $\mathrm{HCN}$ channels differ greatly in different physiological environments, and it is difficult to construct models.

Epilepsy is characterized by the occurrence of spontaneous seizures, i.e., the synchronous discharge of a large number of neurons. In the inherent electrical conductance that governs neuronal excitability, hyperpolarization-activated currents (Ih) play a complex and important role in the fine-tuning of cellular and network activity. Dysregulation of Ih or its conducting ion channel $(\mathrm{HCN})$ is thought to be strongly implicated in epilepsy in humans.

Hyperpolarization-activated cyclic nucleotide-gated ion channels (HCNs) are encoded by four genes (HCN1, $\mathrm{HCN} 2, \mathrm{HCN} 3$, and HCN4). These four isoforms can also be functionally assembled in different combinations to form channels with different properties and structures to conduct Ih currents. Different cells express different types of HCN subunits, and specific neurons express different HCN subunits during development [12-13].HCN channels have very important roles and diverse functions in organisms, with a wide variety of functions in different physiological environments [14].

HCN channels are HCN channels whose specific function depends on interactions with proteins (subunit composition), regulation of neurons and networks, glycosylation, phosphorylation, subcellular distribution, and interactions with other ion channels, among other things. The influence of subcellular distribution on $\mathrm{HCN}$ channel function is reflected in the fact that HCN channels are preferentially localized to the somatic region of the cell for integration and are also present at the axon terminals of some neurons to play a role. Glycosylation, on the other hand, affects the number of HCN channels expressed in the membrane. Phosphorylation of different $\mathrm{HCN}$ channels at multiple sites also affects its biophysical properties. In a study of a rodent model of temporal lobe epilepsy (TLE), it was found that the co-subunit that regulates the cellular distribution of $\mathrm{HCN}$ channels, the tetrapeptide repeat-containing Rab8b interacting protein (TRIP8b), has a phosphate change site at residue Ser237, which enhances the binding of HCN channels. And the channel gating was altered by changing the affinity of TRIP8b for HCN cytoplasm [15].

It follows that because HCN channels have excellent plasticity, they are also functionally diverse. So when the plasticity mechanism of $\mathrm{HCN}$ channels is disrupted or damaged, dysregulation of $\mathrm{Ih}$ can lead to neurological diseases[14].

The Ih current conducted by HCN channels has very specific biophysical properties. First, it is an inward depolarizing current that can correspond to changes in membrane potential and has a depolarizing effect. Secondly, it can also counteract the hyperpolarization of the excited cells.HCN channels are structurally similar to $\mathrm{K}+$ channels, but lack a high selectivity for $\mathrm{K}+$, in fact, they mainly conduct $\mathrm{Na}+$ in the physiological environment,Ih is activated when the cell membrane is relatively hyperpolarized, conducts a reverse potential cationic current, and maintains the stability of neuronal excitation. This feature differs from the classical voltagegated ion channels that increase with depolarization. There is also a higher density of HCN channels in dendrites, and they inhibit or excite action potentials by controlling Ih to control the resistance of the current as it enters the postsynaptic membrane, thereby decreasing or increasing the magnitude of the action potential current input to the postsynaptic membrane. It follows that Ih can cause hyperexcitatory potential depolarization and can also control action potential excitation. In addition, the interaction of cAMP molecules with HCN channels allows for more diverse conductive properties of HCN channels. cAMP can affect the function of $\mathrm{HCN}$ channels by accelerating kinetics and altering voltage activation [16$17]$.

Hyperpolarization

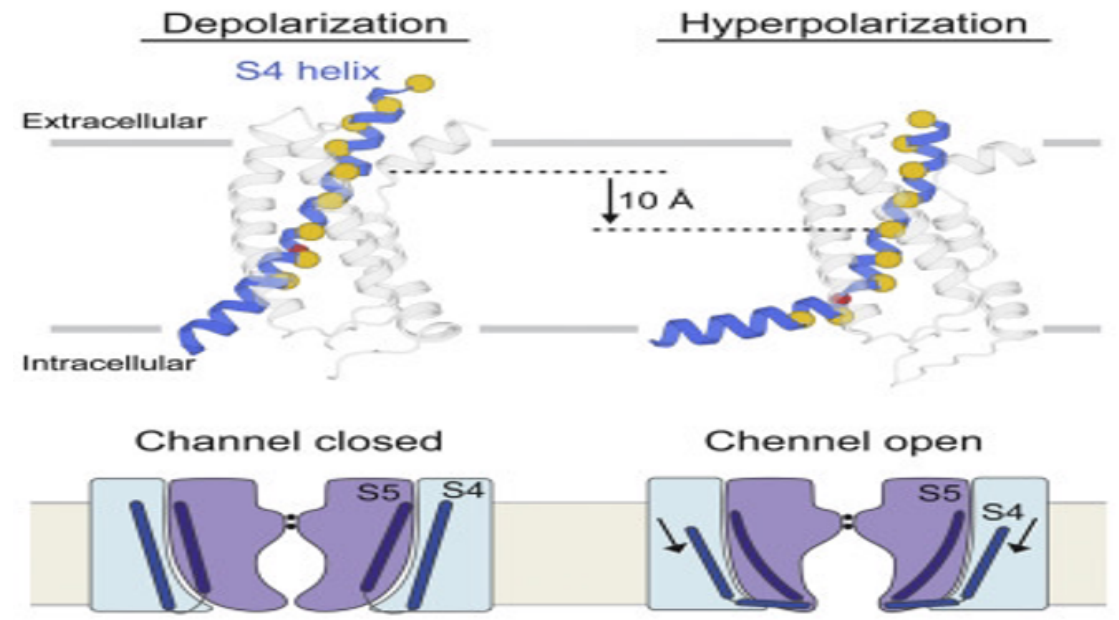

Fig1. HCN channels[18] 
So far, it seems that scientists have achieved some success in studying the function and functional mechanisms of HCN channels. However, there are no specific answers to the questions of how these individual mechanisms combine to function to maintain normal physiological processes in the human or other mammalian physiological environment, or how several abnormal groups trigger the loss of partial function of the entire $\mathrm{HCN}$ channel in the physiological environment, leading to epilepsy.

The above results and discussion can demonstrate a potential link between alterations in HCN channels and Ih current-related functions and the development of epilepsy, but it is undeniable that the current study has not yielded results to prove that the absence or absence of functions of $\mathrm{HCN}$ channels that regulate cellular excitability can directly lead to seizures. This has not been confirmed, including in studies on rodent models. Brennan [15] mentioned in his review several studies on the relationship between HCN1 and HCN2 mutations and epilepsy, none of which could definitively prove that mutations in this gene are a direct cause of epilepsy, only that they are related. In clinical studies, scientists find changes in the function of $\mathrm{HCN}$ channels caused by epilepsy, and also the possibility of the next epileptic episode occurring because of these irreversible damage to HCN channels. The effects of some of the clinically used antiepileptic drugs on Ih could also demonstrate that HCN channels play a role in neuronal excitability and epilepsy. We have already had much work on the molecular mechanisms of HCN channel action, and the study by Chia-Hsueh et al. addressed the voltage channel activation pathway of $\mathrm{HCN} 1$ at the atomic level. They confirmed the hyperpolarized HCN channel model and discovered the mechanism of motion of the S4 helix loop in it[18]. However, there are still many unknown mechanisms and intermolecular relationships, such as the diverse binding modes of different sites among subunits, and the effects of phosphorylation and dephosphorylation of different sites of co-subunits on the regulation of cellular functions by $\mathrm{HCN}$ channels, which will be studied by scientists in the future.

\section{CONCLUSION}

Regarding the pathogenesis of epilepsy, the regulation between molecules, cells, and genes is intricate. The sodium ion channel Nav1.1 and the HCN channel are only two of the many ion channels. As mentioned above, we have achieved some research results on the partial roles and molecular mechanisms of the two ion channels Nav1.1 and HCN channels, but we have never stopped exploring their deeper mechanisms in the past or in the future.

The onset of epilepsy and the connection between them are also being further confirmed. In addition to these two ion channels, several other ion channels related to neuronal excitability have also been suggested to be associated with epilepsy, including potassium ion channels, calcium ion channels, other sodium ion channels, etc. Niday et al. reviewed the important role of potassium ion channels in influencing the excitability of human neuronal networks, and discussed and speculated on the effect of potassium ion channels in epilepsy patients. effects within cells and in neural networks [19]. Valerie et al. found that early $\mathrm{P} / \mathrm{Q}$ calcium channel defects at synapses of a single cell type within the thalamocortical circuit can produce a generalized epileptic phenotype [20].

In addition to the ion channels associated with seizures, various receptors that control neuronal excitability have been a breakthrough in the understanding and treatment of epilepsy. The current research on GABAA receptors is also very hot, scientists are focusing on the subunit composition, gene regulation mode, and molecular mechanism of GABAA receptors, etc., and the obtained research results prove that we are one step closer to dissecting the mechanism of epileptogenesis and optimizing the treatment of epilepsy.

The molecular mechanisms of epileptic seizures will be easier and more accessible to study with advances in technology and equipment. Although the equipment and technology we have to study the molecules have matured, we still face many challenges in resolving the complex mechanism of epileptogenesis, such as how to construct the model and how to exclude the interference of irrelevant factors. Among them there are some difficulties in model building because of the ever-changing forms of dysfunction of ion channels or key molecules in the winning environment of different species. But many of these difficulties are also being addressed, and the unknown future holds infinite possibilities.

\section{ACKNOWLEDHEMENTS}

Thanks for professor Christopher Deppmann and the research program Cetus Online. And thanks to all the professors' results.

\section{REFERENCES}

1. Mulley JC, Scheffer IE, Petrou S, Dibbens LM, Berkovic SF, Harkin LA. SCN1A mutations and epilepsy. Hum Mutat. 2005;25(6):535-542.

2. Escayg A, MacDonald BT, Meisler MH, Baulac S, Huberfeld G, An - Gourfinkel I, Brice A, LeGuern E, Moulard B, Chaigne D, Buresi C \& Malafosse A (2000). Mutations of SCN1A, encoding a neuronal sodium channel, in two families with GEFS+2. Nat Genet 24, 343-345.

3. Claes L, Del - Favero J, Ceulemans B, Lagae L, Van Broeckhoven C\&De Jonghe P (2001). De novo mutations in the sodium - channel gene SCN1A cause severe myoclonic epilepsy of infancy. Am J Hum Genet 68, 1327-1332.

4. Miriam H. Meisler, Jennifer A. Kearney[J]. Sodium channel mutations in epilepsy and other neurological disorders. 2005;115(8):2010-2017.

5. Andrew Escayg, Armin Heils, Bryan T. MacDonald, Karsten Haug, Thomas Sander, Miriam H. Meisler,A Novel SCN1A Mutation Associated with Generalized Epilepsy with Febrile Seizures Plus - and Prevalence 
of Variants in Patients with Epilepsy, The American Journal of Human Genetics, Volume 68, Issue 4,2001,Pages 866-873,ISSN 0002-9297.

6. Miriam H. Meisler, Janelle E. O'Brien, Lisa M. Sharkey, Sodium channel gene family: epilepsy mutations, gene interactions and modifier effects, Journal of Physiology, Volume588, Issue11, June 2010, Pages 1841-1848.

7. Martin MS, Dutt K, Papale LA, Dube CM, Dutton SB, De Haan G, Shankar A, Tufik S, Meisler MH, Baram TZ, Goldin AL \& Escayg A (2010). Altered function of the SCN1A voltagegated sodium channel leads to GABAergic interneuron abnormalities. J Biol Chem 285, 98232834.

8. Xu Haiqing, Cai Xiuqu, Yu Lu, Wang Jie, Liao Weiping, Shi Yiwu, Gao Meimei, Tang Bin. Effects of a truncation mutation in Dravet syndrome-associated NaV1.1 (F1237fsX1269) on the expression and function of sodium channel membrane proteins $[\mathrm{J}]$. Chinese Journal of Neuromedicine|Chin J Neuromed,2015,14(12):1266-1270.

9. Liu Jingxin. Effects of Nav1.1 mutations in neurons of pluripotent stem cell origin induced in epileptic patients and their pathogenesis [D].University of Science and Technology of China,2016.

10. Kang JQ \& Macdonald RL (2009). Making sense of nonsense GABAA receptor mutations associated with genetic epilepsies. Trends Mol Med 15, 430438 .

11. Nancy Osorio, Laurence Cathala, Miriam H. Meisler, Marcel Crest, Jacopo Magistretti, Patrick Delmas, Persistent Nav1.6 current at axon initial segments tunes spike timing of cerebellar granule cells, February 2010, Volume588, Issue4, Pages 651670

12. Brennan, Gary P Baram, Tallie Z Poolos, Nicholas P, Hyperpolarization-Activated Cyclic NucleotideGated (HCN) Channels in Epilepsy., Cold Spring Harbor perspectives in medicine, 6(3), 2016.

13. Brewster AL, Chen Y, Bender RA, Yeh A, Shigemot o R, Baram TZ. 2007. Quantitative analysis and subcellular distribution of mRNA and protein expression of the hyperpolarization-activated cyclic nucleotide-gated channels throughout development in rat hippocampus. Cereb Cortex 17: 702-712.

14. Yoav Noam, Christophe Bernard, Tallie Z Baram,Towards an integrated view of HCN channel role in epilepsy,Current Opinion in Neurobiology,Volume 21, Issue 6,2011,Pages 873879,ISSN 0959-4388.

15. Foote, Kendall M Lyman, Kyle A Han, Ye Michailidis, Ioannis E Heuermann, Robert J Mandikian, Danielle Trimmer, James S Swanson, Geoffrey T Chetkovich, Dane M, Phosphorylation of the HCN channel auxiliary subunit TRIP8b is altered in an animal model of temporal lobe epilepsy and modulates channel function. The Journal of biological chemistry, 294(43), 2019.

16. Biel M, Wahl-Schott C, Michalakis S, Zong X. Hyperpolarization-activated cation channels: from genes to function. Physiol Rev. 2009;89:847-885. A comprehensive review of $\mathrm{HCN}$ channel structure, function and regulation.

17. Robinson RB, Siegelbaum SA. Hyperpolarizationactivated cation currents: from molecules to physiological function. Annual Review of Physiology. 2003;65:453-480.

18. Chia-Hsueh Lee, Roderick MacKinnon, Voltage Sensor Movements during Hyperpolarization in the HCN Channel, Cell, Volume 179, Issue 7, 2019, Pages 1582-1589.e7, ISSN 0092-8674

19. Niday Z, Tzingounis AV. Potassium Channel Gain of Function in Epilepsy: An Unresolved Paradox. The Neuroscientist: a Review Journal Bringing Neurobiology, Neurology and Psychiatry. 2018 Aug;24(4):368-380.

20. Valerie C. Bomben, Isamu Aiba, Jing Qian, Melanie D. Mark, Stefan Herlitze and Jeffrey L. Noebels, Isolated P/Q Calcium Channel Deletion in Layer VI Corticothalamic Neurons Generates Absence Epilepsy,Journal of Neuroscience 13 January 2016, 36 (2) 405-418. 